\title{
Approaches to fatigue life prediction under multiaxial loading
}

\author{
David Nowell ${ }^{1,1}$, and João Vitor Sahadi Cavalheiro ${ }^{2}$ \\ ${ }^{1}$ Imperial College London, South Kensington Campus, London, SW7 2AZ, UK \\ ${ }^{2}$ University of Oxford, Department of Engineering Science, Parks Road, Oxford, OX1 3PJ, UK
}

\begin{abstract}
The paper will consider a set of biaxial experiments, conducted using a cruciform specimen design, manufactured from Waspaloy, a nickel superalloy used in aircraft engine disks. These are analysed using a number of standard, as well as novel, multiaxial fatigue parameters. The results show that most of the existing parameters appear to correlate the results adequately in the region which can be accessed by tension-torsion experiments, but are much less convincing outside this range. A number of potential alternative approaches will be discussed and compared with the experimental results.
\end{abstract}

\section{Introduction}

Many engineering components in service suffer multiaxial loading. Examples include pressure vessels, aircraft engine discs and frictional contact interfaces. Despite this, the designer frequently has to estimate fatigue performance on the basis of uniaxial data only. Multiaxial experiments are expensive, and even then may not simulate the required loading conditions very closely. Since the majority of fatigue cracks in low and high cycle fatigue initiate at the surface (as opposed to the case of very high cycle fatigue), we can reduce the field of practical interest for most applications to that of biaxial loading. Hence a cruciform specimen design might be thought reasonable, and capable of simulating the required loading conditions. However, such experiments are still expensive, and frequently tension-torsion tests are carried out as a cheaper alternative. These, however, are not capable of simulating the complete range of loading conditions, and two quadrants of the principal stress plane are not accessible without internal pressurisation (see Fig.1.). Further, there remains the complication of the type of biaxial loading. At its simplest, the loads may be applied proportionally, and this is clearly the easiest type of loading to consider. Beyond this, the loads may be non-proportional but with fixed principal stress directions. The most complex case is where the principal stress directions vary, as can be the case with frictional contact, such as fretting fatigue experiments.

It is interesting to consider the extent to which the configuration of fatigue experiments is driven by experimental convenience, rather than relevance to the real application.

\section{Corresponding author: d.nowell@imperial.ac.uk}


Certainly there are many industrial applications where the stress field is close to uniaxial: the skin of aircraft wings or the fan blades of a gas turbine, for example. However, there are many which are not: e.g. aircraft engine disks, or pressurised aircraft fuselages. In both of these cases, the stress field produced has a positive biaxiality, which cannot be investigated using the most common type of biaxial test machine, the servo-hydraulic tension-torsion machine. Reports of more complex machines, such as cruciform arrangements [1] or tension/torsion with internal pressure [2], which are more flexible, are relatively rare in the literature. As a consequence, the majority of candidate multiaxial fatigue criteria have been validated primarily against tension/torsion experiments.

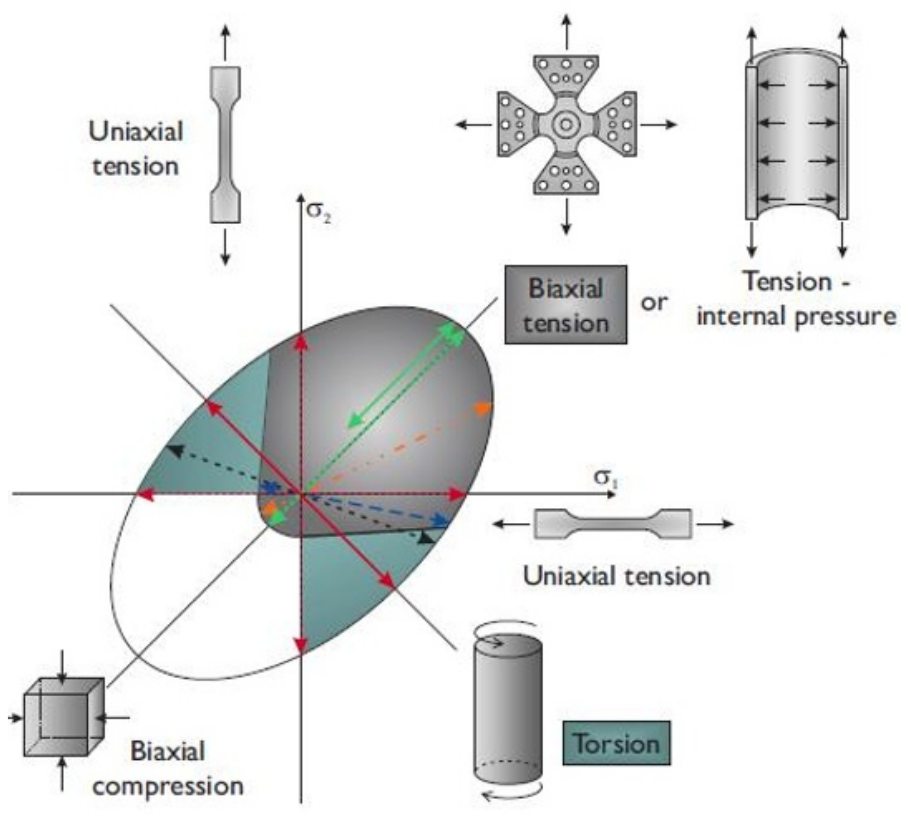

Fig. 1. Different configurations of fatigue experiment, shown in the $\sigma_{1,} \sigma_{2}$ plane.

\section{Experiments}

The experiments were carried out on a three-actuator biaxial testing machine at the University of Oxford [3] An overview of the machine is shown in Fig. 2. Use of three actuators rather than four reduces difficulties with the location of the specimen, but it does mean that long drawbars are required between each of the two horizontal actuators and the specimen grips, in order to reduce the effects of vertical misalignment. 


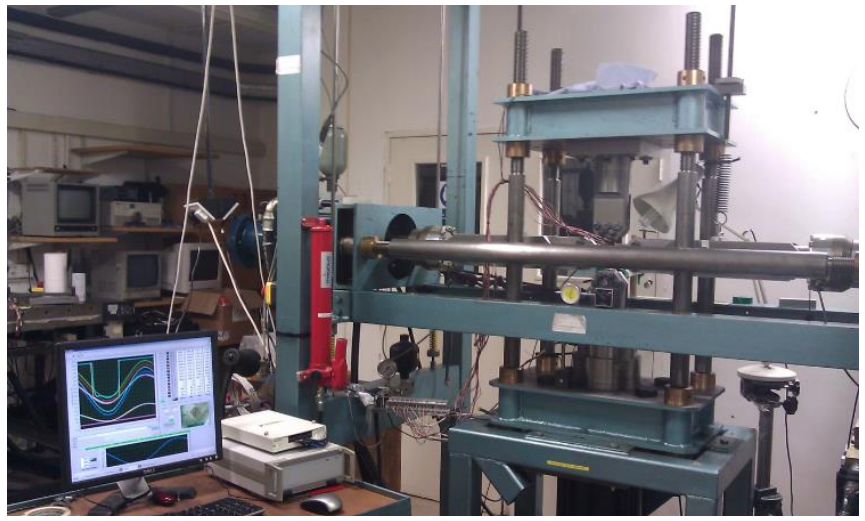

Fig. 2. Biaxial testing machine at the University of Oxford
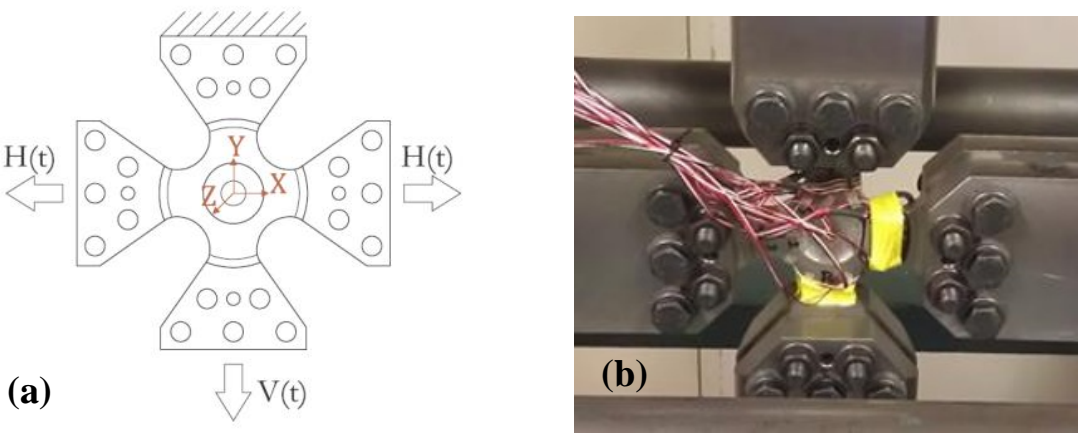

Fig. 3. Cruciform specimen design (a) schematic, (b) mounted in the fatigue machine

The specimens (Fig. 3a.) were made from Waspaloy, a nickel-based aerospace superalloy, widely used in the manufacture of aircraft engine disks. The composition of the alloy is given in Table 1. It has a Young's modulus of $213 \mathrm{GPa}$ and a Poisson's ratio of 0.284. Because of the expense of manufacturing the specimens, only a limited number of experiments could be carried out. The initial series were designed to investigate the effect of a second principal stress on the fatigue life obtained for a given amplitude of the primary principal stress. All of the tests were carried out under proportional loading and with a stress ratio of $\sigma_{\min } / \sigma_{\max }=$ 0.05 . Figure 4 shows the experiments undertaken, with their stress state plotted on the $\sigma_{1}, \sigma_{2}$ plane. Different ratios between the secondary and primary principal stresses were examined by using a finite element model to relate the applied loads to the stresses in the reduced thickness gauge section. These comprised pure shear (-1.0), Uniaxial load (- 0.3), uniaxial stress (0.0), minimum von Mises stress (0.5) and equi-biaxial (1.0). Details of the test conditions and experimental fatigue lives are given in Appendix 1.

Table 1. Nominal chemical composition of Waspaloy.

\begin{tabular}{|c|c|c|c|c|c|c|}
\hline Element & Ni & Cr & Co & Mo & Ti & Al \\
\hline Weight $\%$ & 58 & 19 & 13 & 4 & 3 & 1.4 \\
\hline
\end{tabular}




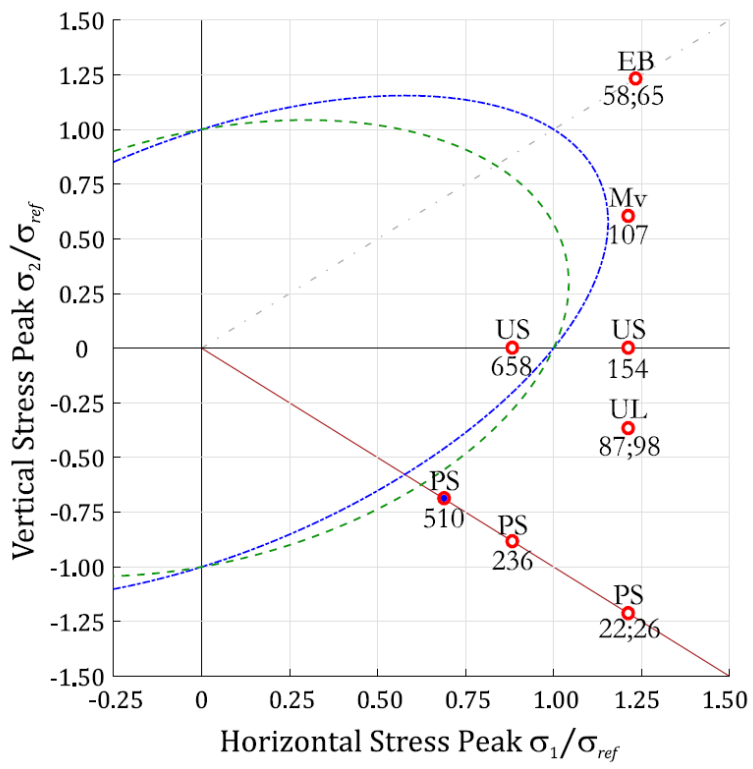

- Test Case

- Runout

nn Cycles 000s

--_von Mises

- - - Strain Energy

-.- Equi-biaxial (EB)

- Pure Shear (PS)

US $=$ Uniaxial Stress

$\mathrm{UL}=$ Uniaxial Load

$\mathrm{MV}=\min$ von Mises

Fig. 4. Experimental conditions plotted on the $\sigma_{1}, \sigma_{2}$ plane

Three additional experiments were carried out, two under pure shear but with lower maximum stress values, and one under uniaxial stress, similarly with a lower maximum value. Co comparing the seven tests undertaken with a normalised peak stress of $1.2^{2}$, we can see that the maximum life seems to occur for the uniaxial stress state. Addition of a nonzero secondary principal stress appears to reduce the life, whether the stress is tensile or compressive. Hence, a life of 154,396 cycles under uniaxial conditions, falls to around 23,000 cycles for pure shear, and 61,000 cycles for equi-biaxial loading. In our previous paper [4], we showed that these results were not consistent with commonly-used multiaxial fatigue parameters. To take a simple example, if we use the amplitude of von-Mises equivalent stress to characterise the effect of the second principal stress on the fatigue performance, we obtain:

$$
\sigma_{e q}=\frac{1}{\sqrt{2}} \sqrt{\left(\sigma_{1}-\sigma_{2}\right)^{2}+\left(\sigma_{2}-\sigma_{3}\right)^{2}+\left(\sigma_{3}-\sigma_{1}\right)^{2}}
$$

For a point on the surface, $\sigma_{3}=0$, and, writing the biaxiality ratio $\sigma_{2} / \sigma_{1}=\Gamma^{3}$, we obtain

$$
\sigma_{e q} / \sigma_{1}=\sqrt{1-\Gamma+\Gamma^{2}}
$$

From this, it is straightforward to derive that for a constant $\sigma_{1}$, the minimum von-Mises stress occurs at a biaxiality ratio of 0.5 , where it is $13 \%$ lower than it is under uniaxial stress. Hence,

\footnotetext{
2 The stress values for these experiments are shown normalised to a reference stress for reasons of commercial confidentiality. ${ }^{3}$ In discussing biaxiality ratio, we must be careful to distinguish between values given by considering load, stress, or strain. In the general case these may all take different values. Indeed, this is the case with our own experiment.
} 
if von Mises stress were thought to be the appropriate parameter to characterise fatigue life, we would expect ratios greater than or less than this to produce shorter fatigue lives than an experiment carried out at this minimum value. Figure 3 plots normalised von Mises stress against biaxiality ratio $\Gamma$. Of course, von Mises stress is hardly a very sophisticated multiaxial fatigue parameter, although it is sometimes used. One can easily envisage stress cycles which follow a constant von Mises equivalent stress, yet which produce fatigue. Similarly, it is possible to envisage a variation in von Mises stress which does not give rise to fatigue failure. However, this example is pertinent, since it shows, in algebraic form, the general behaviour of many commonly used multiaxial fatigue parameters. In this case, a minimum value of the parameter (and hence a maximum fatigue life) is experienced at a non-zero biaxiality ratio. More sophisticated parameters behave in a similar fashion, with many depicting a monotonic increase in life when moving from pure shear to equi-biaxial tension at the same value of $\sigma_{1}$.

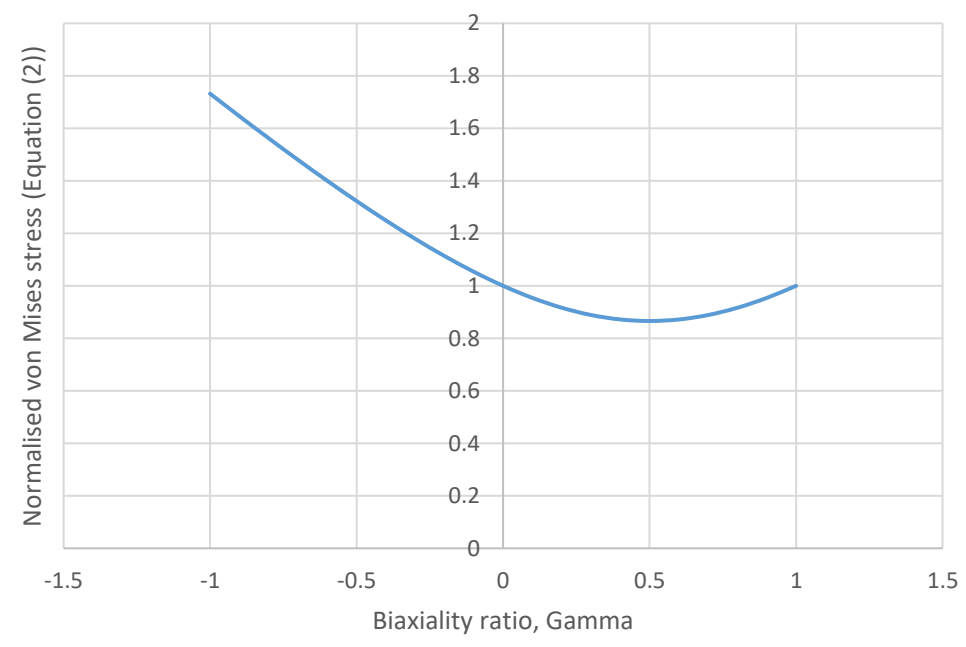

Fig. 5. Variation of normalised von Mises stress with biaxiality ratio $\Gamma$

An alternative means of comparing the experimental results with different multiaxial parameters is shown in Figure 6. Here, the maximum stress state is shown in the hydrostatic (horizontal axis) versus deviatoric (vertical axis) plane. Hence, a state of pure shear lies on the vertical axis. States of uniaxial tension and equi-biaxial tension appear as straight lines in this plane. The experimental results are plotted in the plane, and those carried out at the same value of $\sigma_{l}$ are linked by dotted lines. Also shown in the figure are predictions based on the Crossland criterion [5] of different experimental lives. The expected behaviour from the experimental results is depicted in Figure 7. The longest lives should be obtained for the uniaxial stress condition, and shorter lives should be achieved as we move further away from this line on the diagram. However, the Crossland criterion shows different behaviour. Predicted lives reduce correctly as one moves towards the pure shear axis, but increase as one moves closer to the equi-biaxial tension line. Hence, one concludes that the criterion was formulated to match results accessible in the tension-torsion regime (shown as light grey in the two figures. 


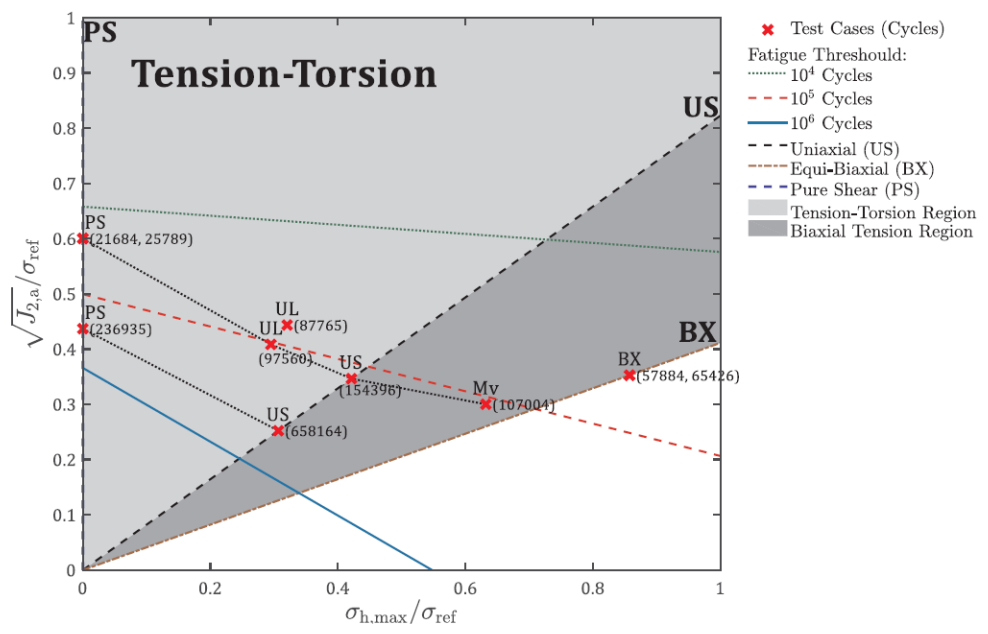

Fig. 6. Comparison of the experimental results with the Crossland criterion.

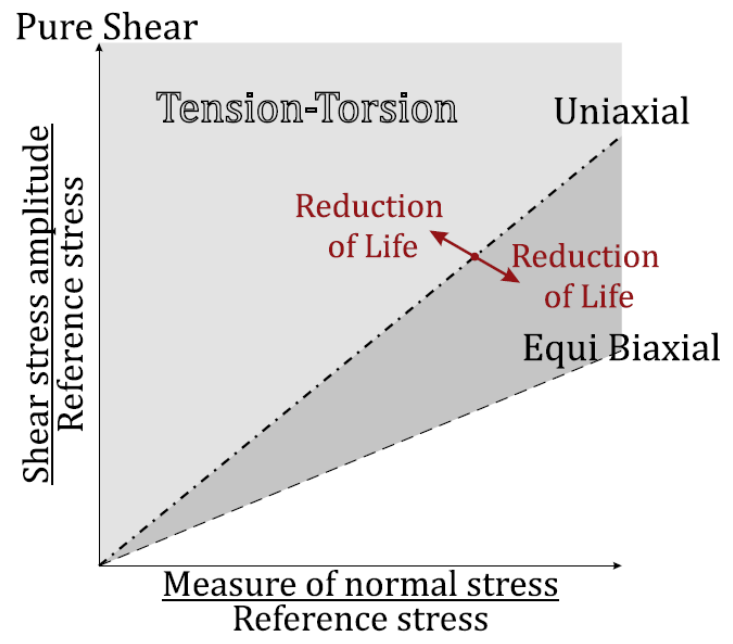

Fig. 7. Expected behaviour in the deviatoric/hydrostatic stress diagram

\section{Conclusions}

The foregoing discussion highlights the fact that commonly used multiaxial fatigue parameters do not capture the behaviour exhibited in our biaxial experiments. More detailed examination of a range of different approaches is described in [3]. One of the key issues is that there is a relative paucity of experimental data available which covers the full range of biaxiality ratio. Hence, it is inevitable that, although there are a large number of multiaxial fatigue parameters available, most of these are calibrated only over a limited range of biaxiality and it is probably feasible to fit quite a number of models to the available data. When these are extrapolated towards the equi-biaxial state, which is only available with 
cruciform tests or internal pressure, they do not necessarily give a satisfactory prediction. Further, we note that most models used are, in essence, empirical curve fits, although they generally start from a physical argument.

Hence, we conclude that existing approaches have limitations, even for the simple case of constant amplitude proportional loading. Recent advances in experimental techniques, such as Electron back-scatter diffraction (EBSD) allow characterisation of the grain structure in polycrystalline materials. This, in turn, may be used to inform crystal plasticity modelling, which may be used to investigate crack initiation [6-8] and small crack growth [9]. This approach is a promising new direction, which may eventually lead us away from current multiaxial criteria. However, careful experimental work will still be needed to validate the model predictions and, though expensive, biaxial cruciform testing has an important role to play in ensuring that a full range of biaxiality is covered in practice.

\section{References}

1. I.H. Wilson and D.J. White, Cruciform specimens for biaxial fatigue tests: An investigation using finite-element analysis and photoelastic-coating techniques, Journal of Strain Analysis, 6(1): 27-37 (1971).

2. D, Lefebvre, C. Chebl, L. Thibodeau and E. Khazzari, A high-strain biaxial-testing rig for thin-walled tubes under axial load and pressure, Experimental Mechanics (1983) 23: 384-392 (1983).

3. J.V. Sahadi, R.J.H. Paynter, D. Nowell, S.J.Pattison and N. Fox, Comparison of multiaxial fatigue parameters using biaxial tests of Waspaloy, International Journal of Fatigue, 100(2): 477-488, 2017.

4. J.V. Sahadi, D. Nowell and R.J.H. Paynter, Fatigue life prediction for Waspaloy under biaxial loading, Theoretical and Applied Fracture Mechanics, 97: 1-14, 2018.

5. B. Crossland, Effect of large hydrostatic pressures on the torsional fatigue strength of an alloy steel, Proc. Int. Conf. on Fatigue of Metals, vol. 138, Institution of Mechanical Engineers London, (1956).

6. K-S. Zhang, J.W. Ju, Z. Li, Y-L. Ba, and W. Brocks, Micromechanics based fatigue life prediction of a polycrystalline metal applying crystal plasticity, Mechanics of Materials, 85: 16-37 (2015).

7. F.P.E. Dunne, Fatigue crack nucleation: mechanistic modelling across the length scales, Curr. Opin. Solid State Mater. Sci., 18: 170-179 (2014).

8. C.A. Sweeney, B. O’Brien, F.P.E. Dunne, P.E. McHugh and S.B. Leen, Strain-gradient modelling of grain size effects on fatigue of CoCr alloy, Acta Mater., 78: 341-353 (2014).

9. H. Proudhon, J.L.F. Wang, A. Roos, V. Chiaruttini and S. Forest, 3D simulation of short fatigue crack propagation by finite element crystal plasticity and remeshing, International Journal of Fatigue, 82(2): Pages 238-246 (2016).

\section{Acknowledgement}

João Sahadi gratefully acknowledges the support of the Brazilian National Council of Technological and Scientific Development (CNPq), Grant No. 207297/2015-0. Specimens for the original biaxial experiments were provided by Rolls-Royce plc. 


\section{Appendix 1 - Experimental Results}

\begin{tabular}{|c|c|c|c|c|c|c|c|c|c|}
\hline \multirow[t]{2}{*}{$\begin{array}{l}\text { Exp. } \\
\text { No. }\end{array}$} & \multirow[t]{2}{*}{ Load case } & \multicolumn{2}{|c|}{ Peak load [kN] } & \multicolumn{2}{|c|}{$\begin{array}{l}\text { Norm. Peak } \\
\text { Strain }\end{array}$} & \multicolumn{3}{|c|}{ Biaxiality Ratio } & \multirow[t]{2}{*}{ Cycles } \\
\hline & & Horiz. & Vert. & $\varepsilon_{x x}$ & $\varepsilon_{\mathrm{yy}}$ & Load & Strain & Stress & \\
\hline CX01 & Single actuator & 117 & 0 & 1.31 & -0.71 & 0 & -0.54 & -0.30 & 87,765 \\
\hline CX02 & Equi-biaxial & 170 & 170 & 0.88 & 0.88 & 1 & 1 & 1 & 65,426 \\
\hline $\mathrm{CX} 03$ & Equi-biaxial & 170 & 170 & 0.88 & 0.88 & 1 & 1 & 1 & 57,884 \\
\hline CX04 & Single actuator & 117 & 0 & 1.31 & -0.71 & 0 & -0.54 & -0.30 & 97,560 \\
\hline CX05 & Pure shear & 90 & -90 & 1.56 & -1.56 & -1.00 & -1.00 & -1.00 & 25,789 \\
\hline CX06 & Pure shear, low $\varepsilon$ & 51 & -51 & 0.88 & -0.88 & -1.00 & -1.00 & -1.00 & $510,000^{*}$ \\
\hline CX07 & Uniaxial Eq. & 128.5 & 38.5 & 1.21 & -0.34 & 0.3 & -0.28 & 0 & 154,396 \\
\hline CX08 & Min von Mises & 147.8 & 102.8 & 1.04 & 0.26 & 0.7 & 0.25 & 0.5 & 107,004 \\
\hline CX09 & $\begin{array}{l}\text { Uniaxial Eq. } \\
\quad \text { low } \varepsilon\end{array}$ & 93.6 & 28.1 & 0.88 & -0.25 & -1.00 & -0.28 & 0 & 658,164 \\
\hline CX10 & Pure shear low $\sigma$ & 65.5 & -65.5 & 1.13 & -1.13 & -1.00 & -1.00 & -1.00 & 236,935 \\
\hline CX11 & Pure shear & 90 & -90 & 1.56 & -1.56 & -1.00 & -1.00 & -1.00 & 21,684 \\
\hline
\end{tabular}

